\title{
Chromatographic and Spectroscopic Fingerprinting of Ficus carica and Evaluation of In Vitro Antioxidant Activity
}

\author{
Amara Javaid, Sobia Anwar, Zahid Ali and Saadia Naseem* \\ Department of Biosciences, COMSATS University Islamabad (CUI), Park Road, Islamabad 45550, Pakistan \\ *For correspondence: saadia.naseem@comsats.edu.pk; saadia1@gmail.com \\ Received 13 November 2020; Accepted 24 December 2020; Published 25 January 2021
}

\begin{abstract}
The present study was conducted to evaluate the in vitro antioxidant activity of Ficus carica, commonly known as fig. Methanol and ethanol extracts of $F$. carica leaves were subjected to 2, 2-diphenyl-1-picryhydrazyl (DPPH) free radical scavenging activity assay where ascorbic acid being positive control had an $\mathrm{IC}_{50}$ value of $3.98 \pm 0.26$ while methanol and ethanol fractions showed an $\mathrm{IC}_{50}$ of $101.76 \pm 1.12$ and $93.12 \pm 1.17$ respectively exhibiting their high antioxidant potential. DPPH assay was also performed on high performance liquid chromatography (HPLC) elutions. Most active antioxidant components in ethanol extract were eluted between 17-18 $\mathrm{min}$, and those in methanol were eluted over 14-15 min and upon ultra-high performance liquid chromatography-mass spectrometery (Orbitrap Liquid Chromatography-Mass Spectrometry) were identified to be 13Docosenamide, $(\mathrm{Z})$ - for ethanol and ficusin for methanol fraction. Thus, it is concluded that these two components are most probable determinants of antioxidant potential of $F$. carica leaf extracts. (C) 2021 Friends Science Publishers
\end{abstract}

Keywords: Ficus carica; Antioxidant; DPPH; HPLC; LC-MS; In vitro

\section{Introduction}

Ficus carica belongs to family Moraceae. It was originated from Western Asia and later spread to the whole world. It is a large shrub to small deciduous tree. F. carica is used traditionally to treat various ailments like gastrointestinal, cardiovascular and respiratory disorders (Duke 2002).

Presence of steroids, coumarins, flavones and triterpenoids in $F$. carica leaves have been reported in plants from European and Asian continents (Peyron et al. 2000; Saeed and Sabir 2002; Vaya and Mahmood 2006). F. carica leaves also exhibits anti-inflammatory, antioxidant (Ali et al. 2012), antimicrobial (Jeong et al. 2009), antidiabetic (Canal et al. 2000), antipyretic (Vikas et al. 2010) and antiHSV activities (Wang et al. 2004). Oxidative stress is one of the main causes of several life threatening neurological and cardiovascular disorders worldwide (Kasote et al. 2015) and the progression of these health issues remains rather unchecked among South-East Asian populations. Nevertheless, the indigenous plants with antioxidant potential can serve as therapeutic agent for all populations ranging from those living in the foothills of the Himalayas to those living in metropolitan zones. Free radical accumulation is responsible for a number of pathophysiological conditions like cardiovascular and neuromuscular disorders. In current era focus is shifted to the use of natural antioxidant compounds for a number of chronic diseases because of their health protecting properties (Teixeira et al. 2009). Antioxidants can reduce or prevent the damage by donation of electron to the damaged cells. Synthetic antioxidants have a number of limitations. They are unstable at high temperatures, and due to their chemical structures, they are volatile, harmful in high doses. Natural antioxidants, on the other hand, are stable at high temperatures, have no impact on the color or odor of the food and have high solubility (Taghvaei and Jafari 2015). So, the search for natural antioxidants is in high demand.

Antioxidant activities of $F$. carica fruit and leaf has been reported in a few studies earlier (Ahmad et al. 2013a; Mawa et al. 2013; Moloudizargari et al. 2013) but the compound/s responsible for the antioxidant activity of $F$. carica were still to be ruled out. So, the specific objectives of our study were to confirm in vitro antioxidant potential of $F$. carica leaf extracts at first and then at next stage our goal was to further perform a comprehensive analysis of the organic extracts of $F$. carica leaves in order to authentically identify compounds responsible for antioxidant potential using latest spectroscopic and chromatographic techniques. It is already well established that a correlation exists between the amount of total phenol and flavonoid and antioxidant capacity of $F$. carica leaves (Konyalığlu et al. 2005). We hypothesized that isolated compounds responsible for strong antioxidant potential of $F$. carica leaf extract may belong either to polyphenols or flavonoids or both of them. So, we aimed to conduct a comprehensive research starting from antioxidant potential of organic 
fractions of $F$. carica leaves and ending up at identifying potential compound responsible for antioxidant potential. If verified by our study it will authenticate the link between presence of polyphenols, flavonoids and antioxidant potential of $F$. carica leaves.

\section{Materials and Methods}

\section{Plant material}

After proper identification, healthy and disease-free leaves of $F$. carica were collected from Plant Genomic Research Institute (PGRI), National Agricultural Research Council (NARC), Islamabad, Pakistan, in March and April 2016. A voucher specimen (FC-AJ-001) was deposited in the herbarium of the Department of Biological Sciences, COMSATS University, Islamabad, Pakistan.

\section{Extraction}

The leaves of $F$. carica were washed gently, yet thoroughly, with deionized water and shade dried at room temperature for two weeks. Sterilized mortar and pestle and a steel grinder were used to grind the dried plants into a fine powder. Ten $\mathrm{g}$ of powdered leaf samples were put in 100 $\mathrm{mL}$ each ethanol and methanol and were sonicated for 30 min. using an ultrasonic cleaning bath (Branson Ultrasonic Cleaner 3210R-DTH, $130 \mathrm{~V}$ input power, frequency of 40 $\mathrm{kHz})$ at $25^{\circ} \mathrm{C}$. Mixtures were then filtered with filtration assembly having a pore size of $0.45 \mu \mathrm{m}$. The filtrate was evaporated under reduced pressure and lyophilized to obtain leaf extracts and were stored at $4^{\circ} \mathrm{C}$ till further use.

\section{Free radical scavenging activity against DPPH reagent}

The solution of 2,2-diphenyl-1-picryhydrazyl (DPPH) radical $(0.2 \mathrm{mM})$ was prepared by mixing $3.94 \mathrm{mg}$ of the DPPH powder to $50 \mathrm{~mL}$ methanol. A 96 well black plate (Corning Incorporated Costar, 3603) was used for the assay. An antioxidant assay was performed according to the protocol of Yang et al. (2011) with minor alterations. Crude extracts were tested for their antioxidant potential. There were three for each sample and twelve standard serial dilutions were prepared for ascorbic acid, and F. carica i.e. $400,300,200,100,50,25,12.5,6.25,3.125,1.5625$, $0.78125,0.39 \mu \mathrm{g} / \mathrm{mL}$. Log of concentration $(\mu \mathrm{g} / \mathrm{mL})$ was plotted against percent DPPH radical scavenging activity. Methanol was used as a negative control. The reaction mixture in each well of 96 well plate consisted of $100 \mu \mathrm{L}$ sample, $100 \mu \mathrm{L}$ DPPH $(0.2 \mathrm{mM})$ in methanol. Experiment was conducted in the dark and absorbance was measured at $517 \mathrm{~nm}$, using a Spectra Max M5 Spectrophotometer (Molecular Devices) after $30 \mathrm{~min}$ incubation. Percent scavenging activity (SA\%) was calculated using the formula (Yang et al. 2011):

$$
\mathrm{SA}(\%)=\frac{A_{517 \mathrm{~nm}}^{C}-A_{517 \mathrm{~nm}}^{S}}{A_{517 \mathrm{~nm}}^{C}} \times 100 \%
$$

Where $A_{517 \mathrm{~nm}}^{C}$ and $A_{517 \mathrm{~nm}}^{S}$ are the absorbance at 517 $\mathrm{nm}$ of the control and a sample, respectively. Graphing and data analysis were carried out with Origin Software. Log of concentration $(\mu \mathrm{g} / \mathrm{mL})$ was plotted against percent DPPH radical scavenging activity. The $\mathrm{IC}_{50}$ values of each raw extract were calculated by fitting the corresponding dose response curve.

\section{High performance liquid chromatography (HPLC) analysis}

Reverse Phase (RP)-HPLC fractionalization of methanol/ethanol extracts was performed on an Agilent 1260 Liquid Chromatography equipped with a quaternary solvent delivery system, an auto-sampler, and a DAD detector. A SHISEIDO CR column $(100 \mathrm{~mm} \times 2.0 \mathrm{~mm})$ was used at an ambient temperature of $25^{\circ} \mathrm{C}$ for all analysis. The mobile phases consist of (A) aqueous formic acid $(0.1 \%$ $\mathrm{v} / \mathrm{v}$ ) and (B) acetonitrile. An elution program was performed using a gradient with a flow rate of $0.2 \mathrm{~mL} / \mathrm{min}$ : 5 to $20 \% \mathrm{~B}$ from 0 to $10 \mathrm{~min}, 20$ to $40 \% \mathrm{~B}$ from 10 to $25 \mathrm{~min}, 40$ to $50 \%$ B from 25 to $40 \mathrm{~min}, 50$ to $60 \% \mathrm{~B}$ from 40 to $45 \mathrm{~min}, 60$ to $70 \% \mathrm{~B}$ from 45 to $58 \mathrm{~min}$ and 70 to $5 \% \mathrm{~B}$ from 58 to $60 \mathrm{~min}$. UV absorption detection was set at $280 \mathrm{~nm}$.

\section{DPPH assay against HPLC fractions}

For both ethanol and methanol extracts, the eluent of ten repeated HPLC separations were collected at each minute for all samples for up to $40 \mathrm{~min}$, and ten fractions at each minute were mixed for lyophilization overnight. Then, each residue was re-dissolved with $100 \mu \mathrm{L}$ methanol subjected to DPPH assay following the procedure described above.

\section{Liquid chromatography and mass spectrometry (LC- MS)}

LC-MS was performed on an Orbitrap Fusion Lumos mass spectrometer (Thermofisher Scientific) coupled to an Ultimate 3000 liquid chromatograph (Thermofisher Scientific). The key parameters for the negative, as well as, positive mode are given in Table 1. Orbitrap LC-MS Data analysis was performed on Xcalibur 4.0 software.

\section{Statistical Analysis}

Software Origin (Pro 8), Origin Lab Corporation, Northampton, MA, USA was used for all statistical analysis. Data were collected from three independent extractions for each fraction and reported as mean \pm standard deviation (SD).

\section{Results}

Free radical scavenging activity of DPPH was evaluated in 1958 (Blois 1958) and today it is the most widely used and reliable method to determine the ability of a compound to 


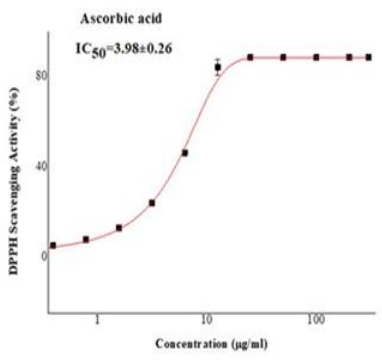

(b)

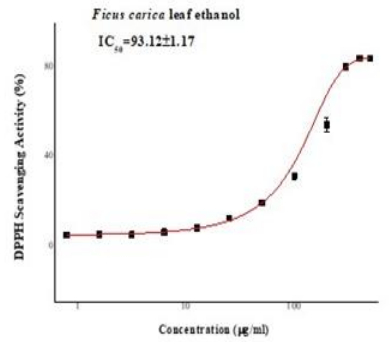

(c)

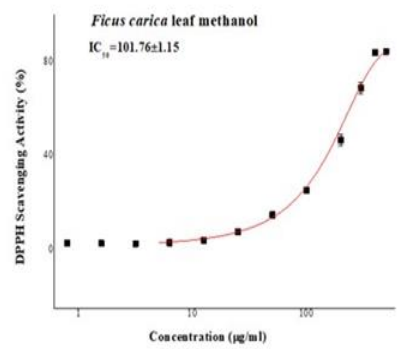

Fig. 1: Antioxidant activity of (a) ascorbic acid (b) ethanol extract and (c) methanol extract of $F$. carica leaves as characterized by DPPH assay $(n=3)$

act as a free radical scavenger and hence possesses an antioxidant potential. $\mathrm{IC}_{50}$ values were calculated by Software Origin (Pro 8), Origin Lab Corporation, Northampton, MA, USA. A sigmoid non-linear regression fitting model was used and a curve was plotted with concentration $(\mu \mathrm{g} / \mathrm{mL})$ along abscissa and percent DPPH scavenging activity was plotted along ordinate. $\mathrm{IC}_{50}$ values of $F$. carica in methanol and ethanol were $93.12 \pm 1.17$ and $101.76 \pm 1.15 \mu \mathrm{g} / \mathrm{mL}$, respectively (Fig. 1b-c) as compared to ascorbic acid i.e. $3.98 \pm 0.26 \mu \mathrm{g} / \mathrm{mL}$ (Fig. 1a). The $\log$ of concentration has been plotted against \% scavenging activity. $\mathrm{IC}_{50}$ below $50 \mu \mathrm{g} / \mathrm{mL}$ was considered high, $\mathrm{IC}_{50}$ between 50 and $100 \mu \mathrm{g} / \mathrm{mL}$ was considered considerably high, between 100 and $200 \mu \mathrm{g} / \mathrm{mL}$ was moderate and little or no activity was considered beyond $200 \mu \mathrm{g} / \mathrm{mL}$.

\section{DPPH assay against HPLC fractions}

In order to identify the bioactive compounds of ethanol and methanol extracts of $F$. carica leaves, we used the DPPH assay described above to evaluate HPLC elutions. Crude methanolic and ethanolic extracts, were subjected to HPLC separately and monitored by UV-Vis detection at $280 \mathrm{~nm}$ (Fig. 2a-b). HPLC fractions were then collected at one min. interval up to $60 \mathrm{~min}$ and the antioxidant activity of each fraction was measured by using DPPH assay in the same manner as performed for crude extracts. As shown in Fig. $2 \mathrm{c}-\mathrm{d}$, the most active components in methanol fraction were eluted between $14-15 \mathrm{~min}$, and those in ethanol fraction eluted over 17 - $18 \mathrm{~min}$.

\section{Liquid chromatography and mass spectrometry (LC- MS)}

Ultrahigh mass resolution mass spectrometry coupled to HPLC (LC-Orbitrap-MS) was utilized to identify the HPLC fractions with potential antioxidants. LC-MS data obtained under both positive and negative ion modes compounds identified in the HPLC fractions are shown in Table 2. According to the DPPH assay and LC-MS data, fraction of methanol eluted between 1-2 min. was identified as methoxsalen and the one eluted between 14-15 min was of
Table 1: LC-MS parameters for positive and negative ion modes for methanol and ethanol fractions of $F$. carica leaves

\begin{tabular}{lll}
\hline Parameters & Positive mode & Negative mode \\
\hline Electrospray voltage $(\mathrm{V})$ & 3000 & 2400 \\
Sheath gas $(\mathrm{Arb})$ & 40 & 40 \\
Auxiliary gas $(\mathrm{Arb})$ & 15 & 15 \\
Sweep gas $(\mathrm{Arb})$ & 2 & 2 \\
Ion transfer tube temp. $\left({ }^{\circ} \mathrm{C}\right)$ & 300 & 300 \\
Vaporizer temp. $\left({ }^{\circ} \mathrm{C}\right)$ & 250 & 250 \\
Detector type & Orbitrap & Orbitrap \\
Orbitrap resolution & 120000 & 120000 \\
Mass range $(\mathrm{m} / \mathrm{z})$ & $150-2000$ & $150-2000$ \\
Maximum injection times $(\mathrm{ms})$ & 50 & 50 \\
AGC target & $4 \mathrm{e} 5$ & $4 \mathrm{e} 5$ \\
RF lens $(\%)$ & 30 & 30 \\
\hline
\end{tabular}

(a)

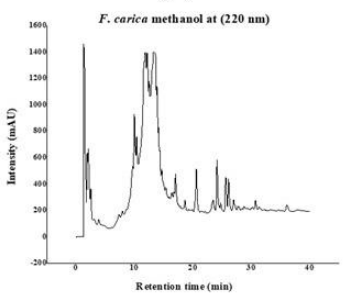

(c)

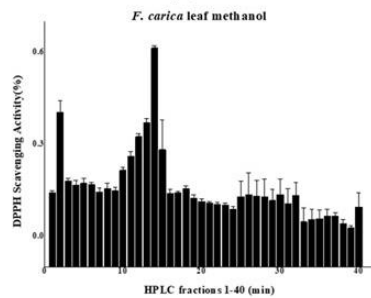

(b)

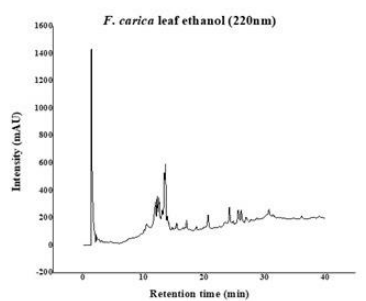

(d)

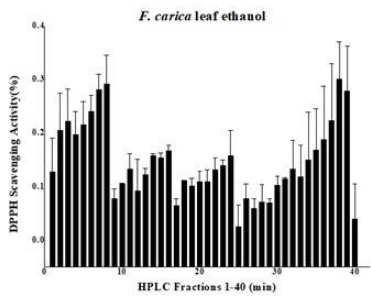

Fig. 2: HPLC Chromatogram detected at $220 \mathrm{~nm}$ of (a) methanol and (b) ethanol extracts of $F$. carica leaves (c, d) antioxidant activity of HPLC fractions of methanol and ethanol extract respectively as measured by DPPH assay

ficusin. Methoxsalen was found in low abundance in methanol extract of $F$. carica leaves as measured by GCMS (Fig. 5b). The most active component in $F$. carica ethanol fraction eluted at 17-18 min was identified to be 13-Docosenamide, $(\mathrm{Z})$ - though it was detected in a low 
Javaid et al. / Intl J Agric Biol, Vol 25, No 3, 2021

Table 2: Components identified in F. carica leaves ethanol and methanol extracts by LC-MS under positive and negative ion modes

\begin{tabular}{lllll}
\hline Retention time $(\min )$ & $m / z\left([\mathrm{M}+\mathrm{H}]^{+} /[\mathrm{M}-\mathrm{H}]^{-}\right)$ & $\mathrm{Z}$ & Mol. formula & Name \\
\hline & & $F$. carica & methanolic extract & \\
$16-17$ & 217.05 & $1+$ & $\mathrm{C}_{12} \mathrm{H}_{9} \mathrm{O}_{4}$ & Methoxsalen \\
$14-15$ & 187.04 & $1+$ & $\mathrm{C}_{11} \mathrm{H}_{7} \mathrm{O}_{3}$ & Ficusin \\
$30-31$ & 279.23 & $1+$ & $\mathrm{C}_{18} \mathrm{H}_{31} \mathrm{O}_{2}$ & $9,12,15$-Octadecatrienoic acid (Z,Z,Z) \\
$1-2$ & 215.03 & $1-$ & $\mathrm{C}_{12} \mathrm{H}_{7} \mathrm{O}_{4}$ & Methoxsalen \\
& & F. carica ethanolic extract & \\
$17-18$ & 338.34 & $1+$ & $\mathrm{C}_{22} \mathrm{H}_{44} \mathrm{O}$ N & 13-Docosenamide, (Z)- \\
$0-1$ & 255.23 & $1-$ & $\mathrm{C}_{16} \mathrm{H}_{31} \mathrm{O}_{2}$ & n-Hexadecanoic acid \\
$0-1$ & 283.26 & $1-$ & $\mathrm{C}_{18} \mathrm{H}_{35} \mathrm{O}_{2}$ & Octadecanoic acid \\
\hline
\end{tabular}

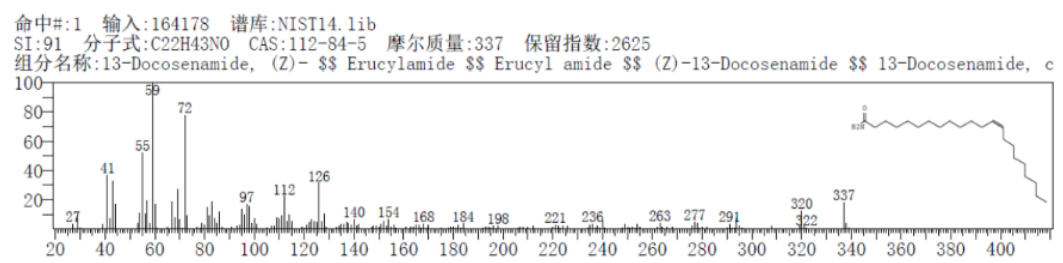

Fig. 3: HPLC Chromatogram of 13-Docosenamide, (Z), found in low abundance but with strong antioxidant potential in $F$. carica leaves
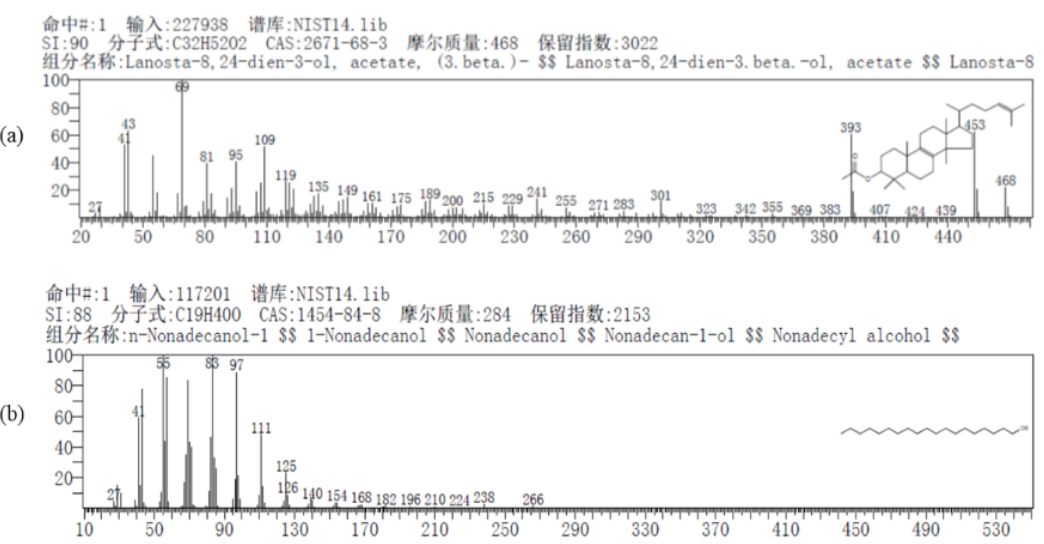

Fig. 4: Chromatograms of (a) lanosta-8,24-dien-3. beta-ol, acetate with RT 50.1min. and peak area14.93\% (b) nonadecanol with RT $21.948 \mathrm{~min}$ and peak area $26.15 \%$. Both compounds were found in high abundance but have low antioxidant potential in $F$. carica leaves

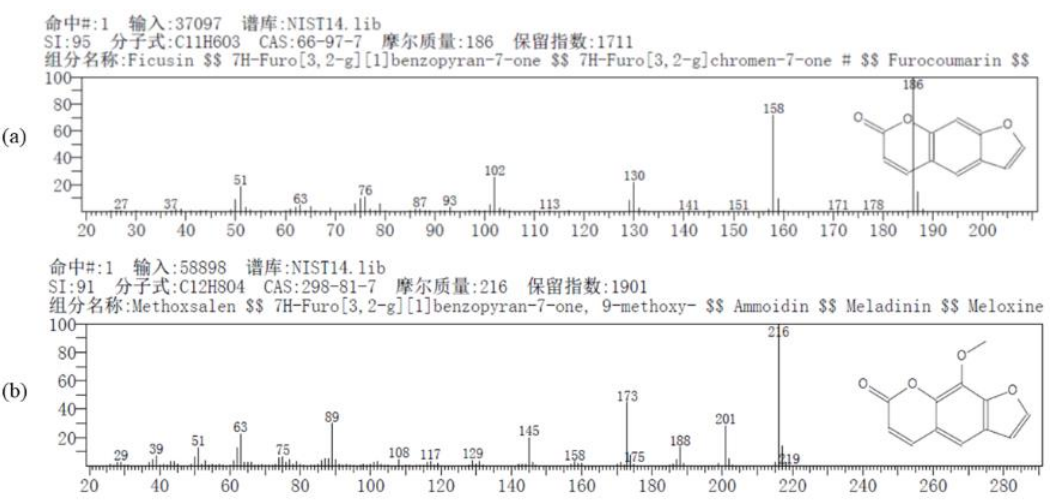

Fig. 5: Chromatogram of (a) ficusin found in low abundance - $0.53 \%$ (b) methoxsalen also in low abundance - $0.44 \%$. Both compounds are strong antioxidant components in $F$. carica leaves

abundance in GC-MS analysis (Fig. 3). So, we concluded that although these compounds are found in lower abundance in methanol and ethanol fractions of $F$. carica leaves, they showed strong antioxidant potential as confirmed by DPPH assay of HPLC fractions and later on via Orbitrap LC-MS. The compound in ethanol fraction with highest abundance as 
detected by GC-MS was lanosta-8, 24-dien-3. beta. -ol, acetate with area under the peak being $14.93 \%$ and retention time $(\mathrm{RT})=50.1 \mathrm{~min}$ (Fig. 4a). Similarly, the compound with highest abundance in methanol extract was nonadecanol with RT 21.948 min and peak area $26.15 \%$ (Fig. 4b). Ficusin is found in low abundance in ethanol fraction $0.53 \%$ (Fig. 5a). Methoxsalen was present in low abundance $0.44 \%$ in methanol fraction (Fig. 5b).

\section{Discussion}

The DPPH is usually used as a reagent to evaluate free radical scavenging activity of antioxidants (Oyaizu 1986). Strong antioxidant potential of $F$. carica leaf has already been reported (Konyalığlu et al. 2005; Mahmoudi et al. 2016). As, mentioned earlier it is known that a correlation is present between amount of total phenol and flavonoid and antioxidant capacity of $F$. carica leaves (Konyalığlu et al. 2005). But an uncertainty was present about the identification of phenol or flavonoid responsible for the antioxidant potential of $F$. carica leaves. In our findings, ficusin found in $F$. carica methanol extract were the main flavonoid responsible for antioxidant potential of $F$. carica. Methoxsalen on the other hand is a furanocoumarin which was also identified in methanol extract of $F$. carica leaf. In a Moroccan study, strong antioxidant potential of $F$. carica was reported (Ayoub et al. 2019), it has also been established that $F$. carica leaves are rich sources of polyphenols at all stages of development (Nadeem and Zeb 2018), possessing strong antioxidant potential. Exhibition of strongest antioxidant potential by ficusin in our study verifies this claim and also shows that our results are comparable to previous reports about $F$. carica being a strong antioxidant candidate and ficusin, being a polyphenol, validate previous studies i.e. F. carica leaves are rich source of polyphenol.

Methanolic extract of $F$. carica leaves also exhibited similar results, having scavenging inhibition of $4.11,8.10$ and $10.22 \%$ at a concentration of 10,150 and $250 \mu \mathrm{g} / \mathrm{mL}$ respectively (Ahmad et al. 2013). A strong antioxidant activity of $F$. carica leaf aqueous-ethanol extract was attributed to the presence of phenolic compounds like methoxypsoralen, rutin, psoralen, dipentoside, dihydoxybenzoic acid and oxypeudacin (Belguith-Hadriche et al. 2017). In another research, $30 \%$ of the total antioxidant activity of $F$. carica leaf, determined via DPPH assay is due to the phenolic compounds present (Teixeira et al. 2009). Strong antioxidant activity of $F$. carica methanol leaf extract was reported in another previous study (Ergül et al. 2019). Our current and previous reports suggest the possible role of $F$. carica leaves in prevention and reduction of free radicals. This potential thus can be translated into a protective role of $F$. carica in chronic diseases.

For herbal drug discovery spectroscopic analysis is the mainstay and hence one of the most important step. This knowledge can lead to the development of new leads which help in designing of new molecules with little modifications. HPLC plays a main role in isolation, separation and fingerprinting of herbal samples and for structure elucidation, mass spectrometry is the technique of the choice. These powerful techniques are revolutionizing the field of natural herbal drug discovery. Thus, the knowledge gained with current study can be used further to design a curative antioxidant drug which is easily accessible, cost effective and has no or fewer side effects. Future perspectives of our study are to determine anti-inflammatory potential of $F$. carica leaves and conduct animal model trails.

\section{Conclusion}

Our study is unique in the aspect that antioxidant compound has been determined in $F$. carica. Results not only validated this information but also gave an insight into the possible antioxidant compounds using Orbitrap LC-MS. The knowledge imparts progress in drug designing with the purpose to introduce the therapeutic remedies for most common ailments through indigenous resources. Identification of specific natural antioxidants from $F$. carica leaves in the present study may give new insights for establishment of biologically derived antioxidants. The folklore usage of this native plant $F$. carica by traditional healers is also justified.

\section{Acknowledgements}

We would like to acknowledge Dr. Mustafa Sajid PGRI (NARC), Islamabad for identification of plant specimen. We are thankful to Higher Education Commission (HEC) of Pakistan for providing partial funding to Amara Javaid, under International Research Support Initiative Program (IRSIP) to conduct part of her Ph.D. research in China. Special thanks goes to research and technical team lead by Prof. Dr. Fuyi Wang at Institute of Chemistry, University of Chinese Academy of Sciences (ICCAS), Beijing, P.R. China. The participating researchers include Wenjuan Zeng, Shumu Li, Yao Zhao and Qun Luo.

\section{Author Contributions}

AJ designed the study, conducted experiments, collected data, performed analytical methods, drafted manuscript. SN conceived the presented idea and supervised the research work. SA and ZA improved write-up. All authors discussed the results and gave critical feedback thus leading to achieve the current form of the manuscript.

\section{References}

Ahmad J, S Khan, D Iqbal (2013) Evaluation of antioxidant and antimicrobial Activity of Ficus carica leaves: an In Vitro approach. J Plant Pathol Microbiol 4:1-4

Ali B, M Mujeeb, V Aeri, SR Mir, M Faiyazuddin, F Shakeel (2012) Antiinflammatory and antioxidant activity of Ficus carica Linn. leaves. Nat Prod Res 26:460-465 
Ayoub L, F Hassan, S Hamid, Z Abdelhamid, A Souad (2019) Phytochemical screening, antioxidant activity and inhibitory potential of Ficus carica and Olea europaea leaves. Bioinformation 15:226-232

Belguith-Hadriche O, S Ammar, MDM Contreras, H Fetoui, A SeguraCarretero, A El Feki, M Bouaziz (2017) HPLC-DAD-QTOF-MS profiling of phenolics from leaf extracts of two Tunisian fig cultivars: Potential as a functional food. Biomed Pharmacother 89:185-193

Blois MS (1958) Antioxidant determinations by the use of a stable free radical. Nature 181:1199-1200

Canal JR, MD Torres, A Romero, C Pérez (2000). A chloroform extract obtained from a decoction of Ficus carica leaves improves the cholesterolaemic status of rats with streptozotocin-induced diabetes. Acta Physiol Hung 87:71-76

Duke JA (2002). Catalog of Herbs, Vol. 2, pp: 300-301. Handbook of medicinal herbs, CRC Press, Boca Raton, Florida, USA

Ergül M, M Ergül, N Eruygur, M Ataş, E Uçar (2019). In vitro evaluation of the chemical composition and various biological activities of Ficus carica leaf extracts. Turk J Pharm Sci 16:401-409

Jeong MR, HY Kim, JD Cha (2009). Antimicrobial activity of methanol extract from Ficus carica leaves against oral bacteria. J Bacteriol Virol 39:97-102

Kasote DM, SS Katyare, MV Hegde, H Bae (2015). Significance of antioxidant potential of plants and its relevance to therapeutic applications. Intl J Biol Sci 11:982-991

Konyalığlu S, H Sağlam, B Kıvçak (2005). $\alpha$-Tocopherol, flavonoid, and phenol contents and antioxidant activity of Ficus carica leaves. Pharm Biol 43:683-686

Mahmoudi S, M Khali, A Benkhaled, K Benamirouche, I Baiti (2016). Phenolic and flavonoid contents, antioxidant and antimicrobial activities of leaf extracts from ten Algerian Ficus carica L. varieties. Asian Pac J Trop Biomed 6:239-245
Mawa S, K Husain, I Jantan (2013). Ficus carica L. (Moraceae) Phytochemistry, traditional uses and biological activities. Evid Based Complem Altern Med, 2013; Article 974256

Moloudizargari M, P Mikaili, S Aghajanshakeri, MH Asghari, J Shayegh (2013). Pharmacological and therapeutic effects of Peganum harmala and its main alkaloids. Pharmacognosy Rev 7:199-212

Nadeem, A Zeb (2018). Impact of maturity on phenolic composition and antioxidant activity of medicinally important leaves of Ficus carica L. Physiol Mol Biol Plants 24:881-887

Oyaizu M (1986). Studies on products of browning reaction. Jpn J Nutr Diet 44:307-315

Vikas P, SC Bhangale, VR Patil (2010). Evaluation of anti-pyretic potential of Ficus carica leaves. Evaluation 2; Article 10

Peyron L, P Pellerin, A Morello, JM Bessiere, A Fruchier, JP Roger (2000). Bergapten content in fig leaves. Ann Falsif l'Expertise Chim Toxicol 93: 427-435

Saeed MA, AW Sabir (2002). Irritant potential of triterpenoids from Ficus carica leaves. Fitoterapia 73:417-420

Taghvaei M, SM Jafari (2015). Application and stability of natural antioxidants in edible oils in order to substitute synthetic additives. $J$ Food Sci Technol 52:1272-1282

Teixeira DM, V Calc, JMG Teixeira, CB Dias (2009). HPLC-DAD quantification of phenolic compounds contributing to the antioxidant activity of Maclura pomifera, Ficus carica and Ficus elastica Extracts. Anal Lett 2986-3003

Vaya J, S Mahmood (2006). Flavonoid content in leaf extracts of the fig (Ficus carica L.), carob (Ceratonia siliqua L.) and pistachio (Pistacia lentiscus L.) BioFactors 28:169-175

Wang G, H Wang, Y Song, C Jia, Z Wang, H Xu (2004). Studies on antiHSV effect of Ficus carica leaves. Zhong Yao Cai 27:754-756

Yang H, Y Dong, H Du, H Shi, Y Peng, X Li (2011). Antioxidant compounds from propolis collected in Anhui, China. Molecules $16: 3444-3455$ 